\title{
Volume Visualization Using Gradient-Based Distance among Voxels
}

\author{
Shinobu Mizuta, Ken-ichi Kanda, and Tetsuya Matsuda \\ Graduate School of Informatics, Kyoto University, 606-8501 Kyoto, JAPAN \\ \{smizuta,kkanda, tetsu\}@i.kyoto-u.ac.jp
}

\begin{abstract}
The aim of this work is to visualize 3D objects in volume data with minimum numbers of user-defined models or parameters. In this report, we propose a novel method that utilizes the distances along the optimum paths between a seed voxel in a target object and other voxels. The distance here is defined using gradient between adjacent voxels along the path. The distance is also used as the criterion of path optimization. The visualization is carried out by rendering the volume where the initial voxel values are replaced with the distances. Experimental results for an image of human embryo obtained with MR microscopy have displayed the effectiveness of this method.
\end{abstract}

\section{Introduction}

Volume visualization is a key technology for the interpretation of 3D images and varieties of methods have been proposed [1]. In the conventional volume rendering techniques, it is quite difficult to observe the target object when the voxel values of an object of interest are similar to others. Meanwhile, most of the methods for the segmentation of target objects from $3 \mathrm{D}$ data are based on user-defined models or parameters, which influence the interpretations of resulting images. The aim of our research is to visualize $3 \mathrm{D}$ objects in volume data with minimum numbers of userdefined models or parameters to comprehend the meaning of images directly.

\section{Methods}

The procedure of volume visualization in the proposed method is as follows: (i) setting a seed voxel in a target object in input 3D volume data, (ii) calculating the distances between the seed voxel and all other voxels along the optimum paths based on Dijkstra algorithm [2], (iii) replacing the initial voxel values with the calculated distances, (iv) rendering the replaced volume. The optimization of paths between voxels is carried out by minimizing "roughness", which is defined by the largest value of absolute gradient between adjacent voxels along a path. The value is denoted by $\mathrm{d}$ $(\mathrm{S}, \mathrm{v}, \mathrm{P})$ for a path $\mathrm{P}$ between the seed voxel $\mathrm{S}$ and another voxel $\mathrm{v}$. The path $\mathrm{P}$ is regarded as "smooth" when $d(S, v, P)$ is small. The minimum value of $d(S, v, P)$ for various $\mathrm{P}$ is denoted by $\mathrm{D}(\mathrm{S}, \mathrm{v})$, where the corresponding path is optimum. $\mathrm{D}(\mathrm{S}, \mathrm{v})$ is used as the distance between the voxels $\mathrm{S}$ and $\mathrm{v}$. The outline of the procedure is shown in Figure 1 (a).

W. Niessen and M. Viergever (Eds.): MICCAI 2001, LNCS 2208, pp. 1197-1198, 2001.

(C) Springer-Verlag Berlin Heidelberg 2001 

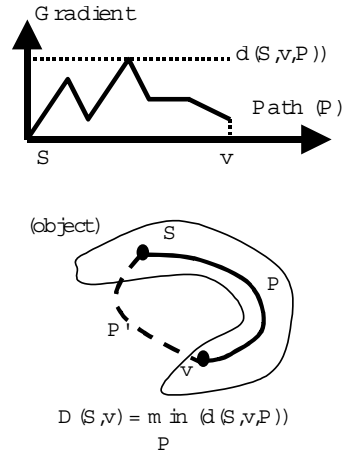

(a)

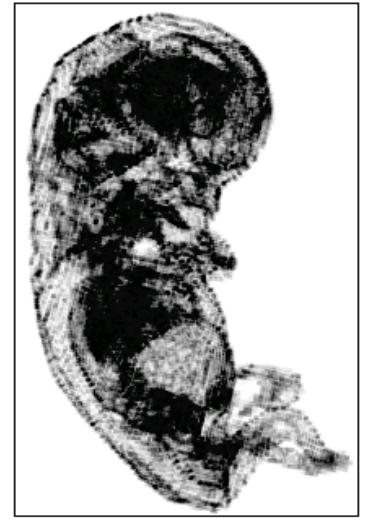

(b)

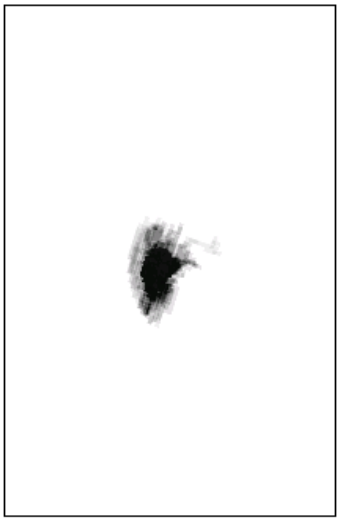

(c)

Fig. 1. (a) Outline of the proposed method. (b) Volume rendering result using conventional method (differences of voxel values from the seed voxel). (c) Volume rendering result using proposed method.

The procedure of finding optimum paths is similar as that of the region growing method for image segmentation [3] or "live-wire" method for interactive search of boundaries [4]. A unique point of our method is calculating the distances along the optimum paths for all voxels in order to utilize them to visualize volumes.

\section{Results}

A 3D image of a human embryo obtained with MR microscopy have visualized by the conventional and proposed methods. The target object is the lung area. The results are shown in Fig.1 (b)(c). By the proposed method, only the target object was visualized as the gradient-based distances from the seed point in the target object.

\section{Acknowledgement}

We would like to thank to K. Shiota and T. Nakatsu for the use of their specimens.

\section{References}

1. Sonka et al., "Handbook of Medical Imaging", SPIE Press, Bellingham, Washington (2000)

2. Cormen et al., "Introduction to Algorithms", McGraw-Hill, New York, NY (1990)

3. Zukker, Comput. Graph. Image Process., vol.5, no.3, pp.382-399 (1976)

4. Mortensen and Barrett, Proc. SIGGRAPH '95, pp.191-198 (1995) 